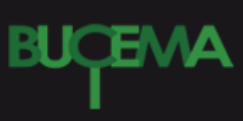

Bulletin du centre d'études médiévales d'Auxerre | BUCEMA

Hors-série $n^{\circ} 6 \mid 2013$

Autour du cloître : les chapelles Notre-Dame et les accès au chapitre

\title{
La chapelle mariale du monastère de Souvigny: Notre-Dame de l'infirmerie ou des avents - entre chapitre et infirmerie
}

Pascale Chevalier, Arlette Maquet et Laurent Fiocchi

\section{(2) OpenEdition}

\section{Journals}

Édition électronique

URL : https://journals.openedition.org/cem/12849

DOI : $10.4000 /$ cem.12849

ISSN : 1954-3093

Éditeur

Centre d'études médiévales Saint-Germain d'Auxerre

Référence électronique

Pascale Chevalier, Arlette Maquet et Laurent Fiocchi, «La chapelle mariale du monastère de

Souvigny : Notre-Dame de l'infirmerie ou des avents - entre chapitre et infirmerie », Bulletin du centre d'études médiévales d'Auxerre / BUCEMA [En ligne], Hors-série nº 6 | 2013, mis en ligne le 29 mars 2013, consulté le 03 mars 2023. URL : http://journals.openedition.org/cem/12849 ; DOI : https://doi.org/ 10.4000/cem.12849

Ce document a été généré automatiquement le 3 mars 2023.

Creative Commons - Attribution - Pas d'Utilisation Commerciale - Partage dans les Mêmes Conditions 4.0 International - CC BY-NC-SA 4.0

https://creativecommons.org/licenses/by-nc-sa/4.0/ 


\title{
La chapelle mariale du monastère de Souvigny : Notre-Dame de l'infirmerie ou des avents - entre chapitre et infirmerie
}

\author{
Pascale Chevalier, Arlette Maquet et Laurent Fiocchi
}

Situé à mi-pente d'une colline argilogréseuse qui s'élève au-dessus d'une petite rivière, la Queune, affluent de la rive gauche de l'Allier, à $13 \mathrm{~km}$ à l'Ouest de Moulins, le prieuré Saint-Pierre de Souvigny appartient aux premières dépendances de Cluny. Il occupe le centre $\mathrm{du}$ bourg monastique qui constitue aujourd'hui la ville de Souvigny. Ce prieuré clunisien se trouvait à l'extrémité nord du diocèse de Clermont, aux limites de ceux de Bourges au nord-ouest, de Nevers au nord-est et d'Autun à l'est, dans une situation de marge, de frontières,

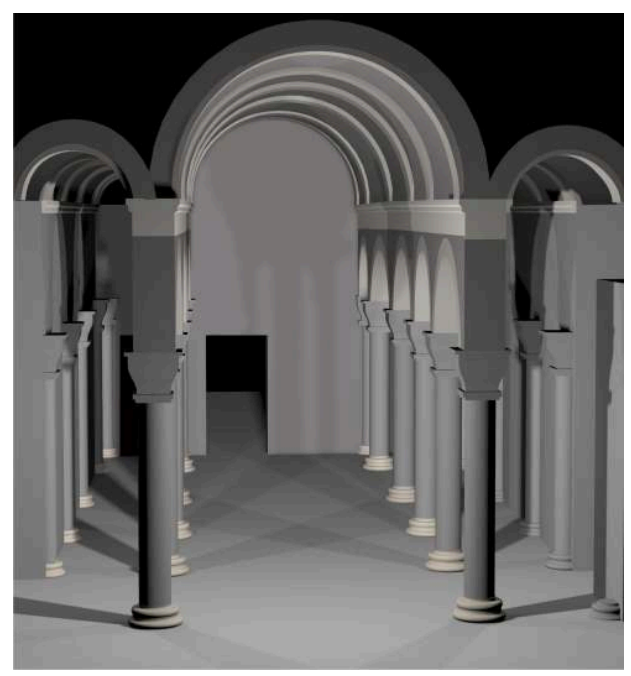
jusqu'à la création de l'évêché de Moulins au XIXe siècle.

2 Après des découvertes suscitées essentiellement par les travaux de confortement et consolidation des édifices, suivis de quelques sondages préventifs limités, plusieurs opérations archéologiques programmées ont été réalisées sur le site de Souvigny à partir de 2001 : un sondage a d'abord permis la découverte du tombeau des saints abbés Mayeul et Odilon de Cluny (2001), puis l'élargissement à la travée a mis au jour les aménagements successifs réalisés autour de celui-ci (2001-2003) ${ }^{1}$. Une fouille (2006-2007) a été menée en aire ouverte dans le cadre d'une restauration portée par les 
Monuments historiques, dans les cinq vaisseaux de la nef ${ }^{2}$ de la priorale, dont le chantier de construction a fait l'objet d'un mémoire de Master $2{ }^{3}$. En parallèle, au cours de la période 2003-2005, une série d'examens a porté sur un autre secteur du site.

Fig. 1 - Vue de la priorale depuis le cloître, vestiges de l'ancienne salle capitulaire et sacristie à l'emplacement de l'église mariale.

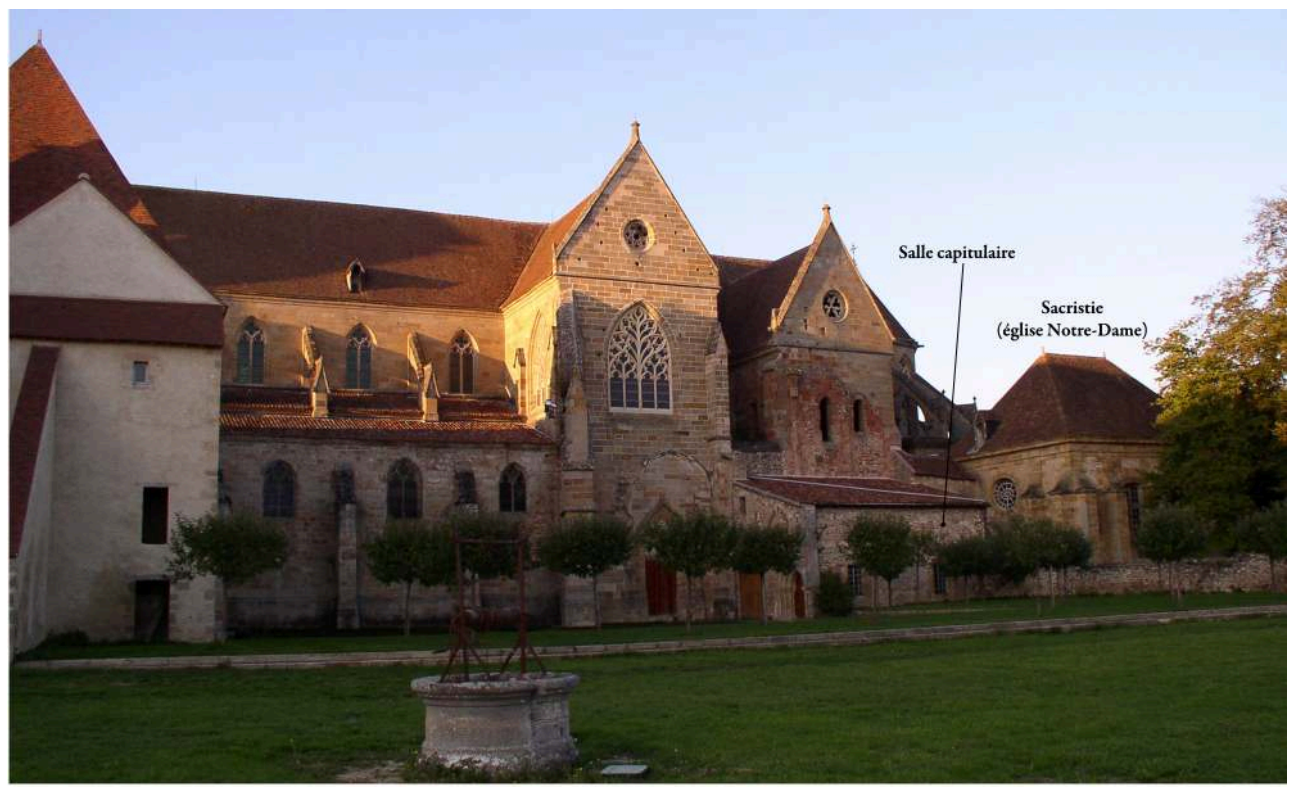

Lors d'un sondage programmé, une partie de l'église Notre-Dame-des-Avents ${ }^{4}$ a été découverte sous la sacristie reconstruite à la fin $d u$ XvIII $^{\mathrm{e}}$ siècle par l'architecte Joseph Évezard (1711-1783) ${ }^{5}$ (fig. 1). L'approfondissement de la fouille a montré la présence sous cette église d'une, puis de deux absides correspondant à des édifices antérieurs (chapelle Sainte-Marie puis Notre-Dame de l'infirmerie) (fig. 2), qui composent au cours du temps le pôle marial du monastère de Souvigny. 
Fig. 2 - Plan des fouilles de 2003-2004 (DAO D. Vuillermoz).

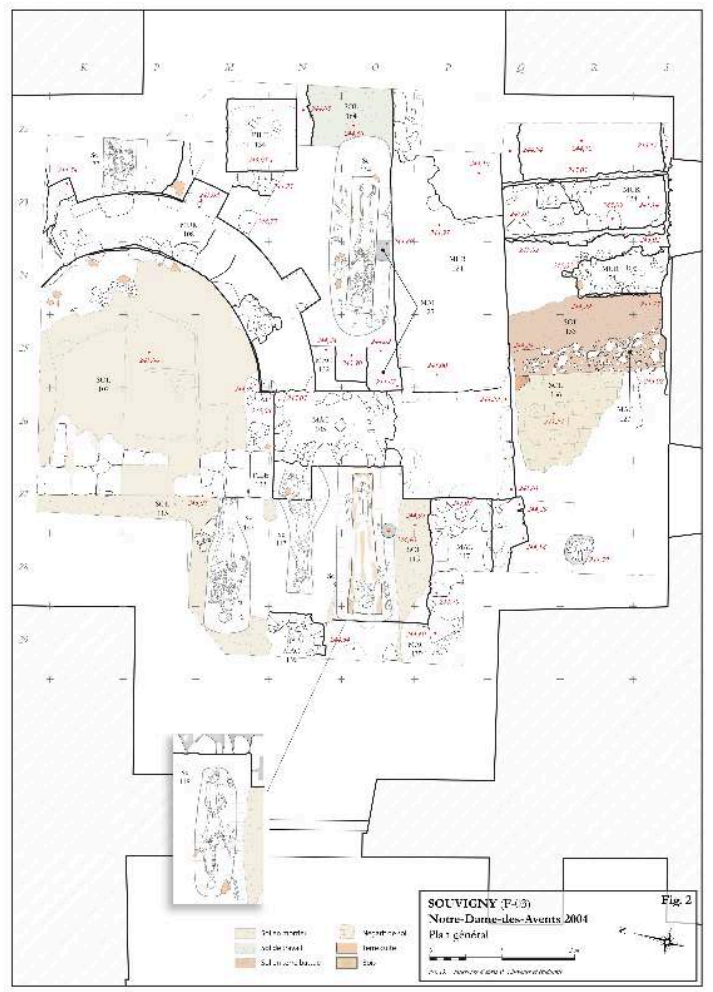

\section{Rappel historique}

4 Le 11 mai 994, Mayeul, le quatrième abbé de Cluny, meurt à Souvigny. C'est là qu'en 915 ou 920, Aymar, le premier ancêtre connu de la famille de Bourbon, a établi en faveur de Cluny une donation, constituée d'une villa avec une église dédiée à saint Pierre et divers biens fonciers (casales, vignes, champs, prés) ${ }^{6}$. Le développement du domaine ne commence qu'à l'installation effective de la communauté monastique au milieu du $\mathrm{x}^{\mathrm{e}}$ siècle $^{7}$, ce qui correspond à l'expansion clunisienne en Auvergne ${ }^{8}$ et dans d'autres régions ${ }^{9}$.

994, Mayeul est enterré devant l'autel de la Croix dans l'église Saint-Pierre que les moines viennent de reconstruire ${ }^{10}$. Son culte, celui du premier abbé de Cluny à être déclaré saint, connaît un essor rapide, ainsi que le rapporte Raoul Glaber, qui place vers 1047-1048 le pèlerinage de Souvigny au même niveau que celui de Saint-Martin de Tours ${ }^{11}$.

6 Son successeur, Odilon, qui a institué vers 1030-1033 la Fête des Morts, célébrée le 2 novembre, et élaboré l'idéologie de la mémoire des défunts entretenue par les prières des moines, est aussi inhumé à Souvigny où il décède le $1^{\mathrm{er}}$ janvier 1049. Désormais, Souvigny détient un rare privilège, celui de posséder les sépultures de deux abbés de Cluny : l'église Saint-Pierre devient un véritable reliquaire. 


\section{L'église majeure - Saint-Pierre - dans la seconde moitié du $x^{\mathrm{e}}$ siècle}

7 Lors de l'arrivée des frères de Cluny à Souvigny sous l'abbatiat de Mayeul, des bâtiments de la villa les accueillent tant d'un point de vue cultuel que fonctionnel. Dans ce nouveau cadre clunisien, les moines entreprennent aussitôt d'agrandir l'église carolingienne de la donation. L'installation en batterie de plusieurs grands fours à chaux apparait comme le signe d'un chantier important. On ignore tout d'un possible transept et du chevet, dont l'emplacement doit correspondre à la croisée du futur grand transept, mais des éléments de la nef ont été reconnus. Un bâtiment rectangulaire carolingien appartenant à la villa conditionne l'orientation du gouttereau sud, son pendant nord remploie celui de l'ancienne église ${ }^{12}$. La grande nef charpentée, légèrement trapézoïdale du fait de ce remploi, mesure en moyenne dans l'œuvre 13,67 x 29,75 m (longueur restituée) ${ }^{13}$, elle est encore caractéristique de l'architecture carolingienne. La façade occidentale, arasée, offre un portail central dont les piédroits en pierre de taille de grès brun cantonnent une ouverture large de 3,22 m. Le seul autre accès connu est, à l'extrémité du gouttereau sud, une porte associée à un sarcophage de seuil. Le déroulement de la construction se déduit de la chronologie des fours à chaux et de leur relation avec le mur de façade. Leur datation par $\mathrm{C}^{14}$ situe le début des travaux au milieu du $\mathrm{x}^{\mathrm{e}}$ siècle. La campagne s'achève par la façade ouest qui vient perturber ces fours. Tout suggère un enchâssement du premier édifice assurant la pérennité du lieu du culte pendant les travaux, puis sa démolition à leur achèvement.

\section{L'église mineure de la bienheureuse Vierge Marie (fin du $\mathrm{X}^{\mathrm{e}}$-début $\mathrm{XI}^{\mathrm{e}}$ siècle ?)}

8 La liturgie clunisienne est de tradition carolingienne, ce qui augure la présence de plusieurs églises dans le complexe monastique. Deux bâtiments fonctionnent assurément avec la priorale Saint-Pierre dans la seconde moitié du $\mathrm{x}^{\mathrm{e}}$ siècle: la construction carolingienne déjà évoquée, au sud de l'église, dont on ignore l'emprise et les divisions éventuelles et un lambeau d'un autre édifice de culte qui peut lui être associé - l'abside de la chapelle « de la bienheureuse Vierge Marie » ${ }^{14}$. Deux textes de la première moitié du XI $\mathrm{x}^{\mathrm{e}}$ siècle rapportent l'agonie à la fin de décembre 1048, de l'abbé Odilon, qui y est transporté à plusieurs reprises. En 2004-2005, un sondage a mis au jour deux églises romanes successives: Notre-Dame de l'Infirmerie et Notre-Dame-desAvents. Le chevet de la première semble entourer l'abside - plus petite, également en hémicycle - d'une chapelle primitive ${ }^{15}$ qui, associée à la priorale de Mayeul, rappelle la disposition duelle de Cluny II. La datation de cette abside (diam. restitué hors œuvre 3,50-3,60 m), assurément antérieure au troisième tiers $\mathrm{du}_{\mathrm{XI}}^{\mathrm{e}}$ siècle, est hypothétique, mais elle est issue d'une réflexion régressive touchant l'ordre perceptible dans les campagnes de construction souvignissoises des $\mathrm{XI}^{\mathrm{e}}$ et $\mathrm{XII}^{\mathrm{e}}$ siècles, qui débutent par la priorale et progressent d'ouest en est, des bâtiments claustraux jusqu'à l'église de l'infirmerie et des morts ${ }^{16}$. Compte tenu de l'estimation des temps de construction et de ce sens ouest/est des chantiers, la chapelle doit exister avant la reconstruction du chœur de la priorale par Odilon (fig. 3) ; par déduction, on intégrera cette abside au projet d'élaboration du prieuré sous Mayeul. La porte sud de la nef de Saint-Pierre 
donnerait alors accès à un espace mal défini (le cloître ?) et à une petite église mariale édifiée autour de l'an mil. Son rôle de chapelle des morts est attesté dès le $\mathrm{XI}^{\mathrm{e}}$ siècle. Sébastien Marcaille ${ }^{17}$, qui écrit au début du XvII ${ }^{\mathrm{e}}$ siècle, présente un épisode miraculeux de la vie d'odilon qui semble spécifique à Souvigny: la tentation de l'abbé par la comtesse Ermengarde de Saint-Maurice. Le saint se jette dans un grand feu pour résister aux avances de la dame. Reconnaissant son erreur, celle-ci demande à être inhumée au chapitre et à bénéficier d'un anniversaire dans la " chapelle Notre-Dame ", avec les sept psaumes pénitentiels de David ${ }^{18}$ (ce service devait avoir lieu tous les ans le dixième jour de l'Avent) et que sa servante soit inhumée dans le cloître.

\section{La priorale Saint-Pierre du $\mathrm{XI}^{\mathrm{e}}$ siècle, les travaux d'Odilon (994-1048) (fig. 3)}

Fig. 3 - Proposition de restitution de la priorale, de la première chapelle mariale et du cloître dans la $1^{\text {ère }}$ moitié du XI ${ }^{\text {e }}$ siècle (DAO L. Fiocchi).

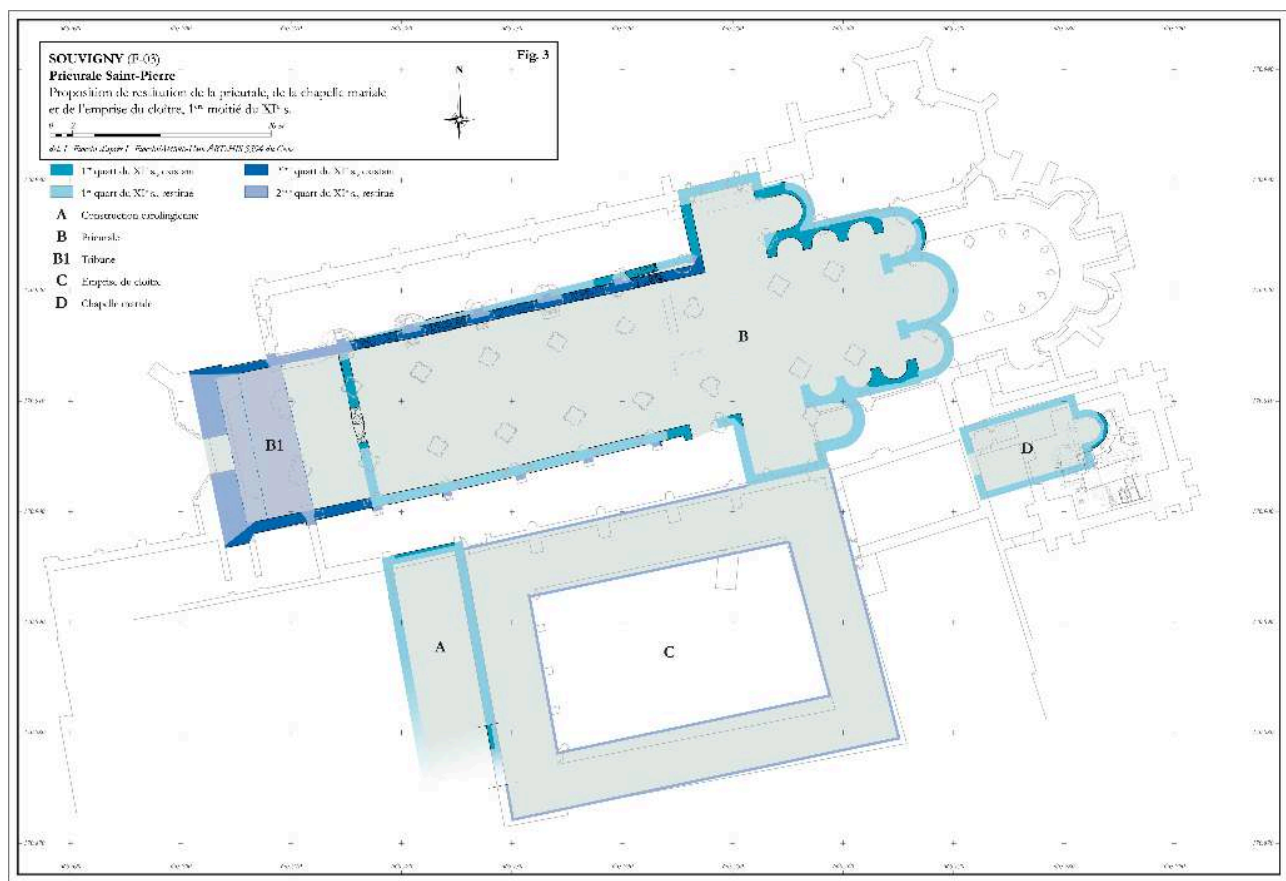

Le poème de Jotsald ${ }^{19}$ évoque la grande priorale d'Odilon. Dès la mort de Mayeul, en 994, des miracles ont lieu et un pèlerinage se met rapidement en place dans l'église juste achevée, mais dont le changement de fonction et la nouvelle dimension d'église de pèlerinage entraînent aussitôt de nouveaux besoins. Ceci ne fait pas de Souvigny un cas isolé, le développement des églises parallèlement à l'essor d'un pèlerinage s'observe aussi dans nombre d'édifices bourguignons ${ }^{20}$.

En 1998, Christian Sapin a remarqué la similitude des plans à nef unique de Paray-leMonial et de Souvigny, le développement du chœur et les absidioles orientées du transept les apparentant au plan de Cluny II ${ }^{21}$. Les vestiges dégagés en $1923^{22}$, complétés par un nouveau relevé en 2006-2008, permettent de restituer un chevet à triple abside assez original, dont les parois nord et sud sont rythmées par trois niches semi-circulaires. Ce chevet aux dimensions comparables à celles de Cluny II ${ }^{23}$, est 
contemporain du transept à profondes absidioles. Les comparaisons suggèrent de le dater du premier quart du XI ${ }^{\mathrm{e}}$ siècle.

11 Odilon amplifie d'abord le chœur architectural et liturgique, témoignant de l'accroissement rapide de la communauté, avant de rebâtir vers l'ouest, au début du deuxième quart $\mathrm{du} \mathrm{XI}^{\mathrm{e}}$ siècle. La position du chœur est centrée sur le vaisseau de Mayeul, alors qu'un décalage de 1,10 $\mathrm{m}$ existe avec la nouvelle nef $\mathrm{du} \mathrm{XI}^{\mathrm{e}}$ siècle, longue de 43,70 m pour 15,10 $\mathrm{m}$ de largeur, éclairée par six paires de grandes baies latérales, très haut percées. Le mur de façade en pierres de taille mesure $3 \mathrm{~m}$ d'épaisseur à sa base et un espace profond de $8,35 \mathrm{~m}$, façade incluse, ne possède aucune baie. Ces derniers éléments suggèrent la présence d'une tribune charpentée aveugle, antérieure à l'édification des tours, accessible par un escalier ménagé dans l'épaisseur de la façade. Si le vaisseau unique est d'esprit carolingien, le plan du chevet à exèdres et la disposition d'un escalier à l'intérieur du mur sont plutôt issus de la tradition antique romaine ${ }^{24}$ - ces emprunts formels relient encore un peu plus Cluny à Rome, à travers Souvigny.

En 1063, le cardinal Pierre Damien procède à la translation du corps du saint abbé Odilon, de son tombeau primitif dans un nouveau monument au centre du vaisseau, et à la consécration de l'église. L'agencement triparti de la nef alors projeté impose pratiquement ce transfert lié aux prémices d'un nouveau chantier. Ainsi se perçoit la continuité du pèlerinage actif, en pleine expansion. La division de la nef en trois vaisseaux voûtés est accompagnée par celle du chœur avec un voûtement du sanctuaire, ainsi que par une tour lanterne à la croisée du transept. Le voûtement des bas-côtés oblige à réduire les fenêtres originelles et à y insérer des baies plus petites. La tripartition est rendue lisible à l'extérieur par la reprise de la façade occidentale, où deux tours d'angle reprennent l'emprise de la tribune de l'état antérieur. On y trouve trois chapelles hautes, formant un véritable avant-corps occidental de tradition carolingienne et ottonienne: une chapelle centrale Saint-Michel, flanquée par deux autres plus petites, dédiées aux archanges.

13 Avec une originalité certaine, l'avant-nef à trois vaisseaux voûtés, ajoutée dans le dernier quart $\mathrm{du} \mathrm{XI}{ }^{\mathrm{e}}$ siècle, est à la fois dépourvue d'étage et dotée de tours à l'est : ces tours sont en effet encore en construction lorsque l'on décide d'édifier la galilée et la préexistence du culte de saint Michel justifie l'absence du second niveau, qui lui est consacré habituellement.

\section{L'église de Notre-Dame l'infirmerie (troisième quart du $\mathrm{Xl}^{\mathrm{e}}$ siècle) (fig. 4)}


Fig. 4 - Proposition de restitution de la priorale, de la chapelle Notre-Dame de l'infirmerie et du cloître dans le $3^{\text {ème }}$ quart du $\mathrm{Xl}^{\mathrm{e}}$ siècle (DAO L. Fiocchi).

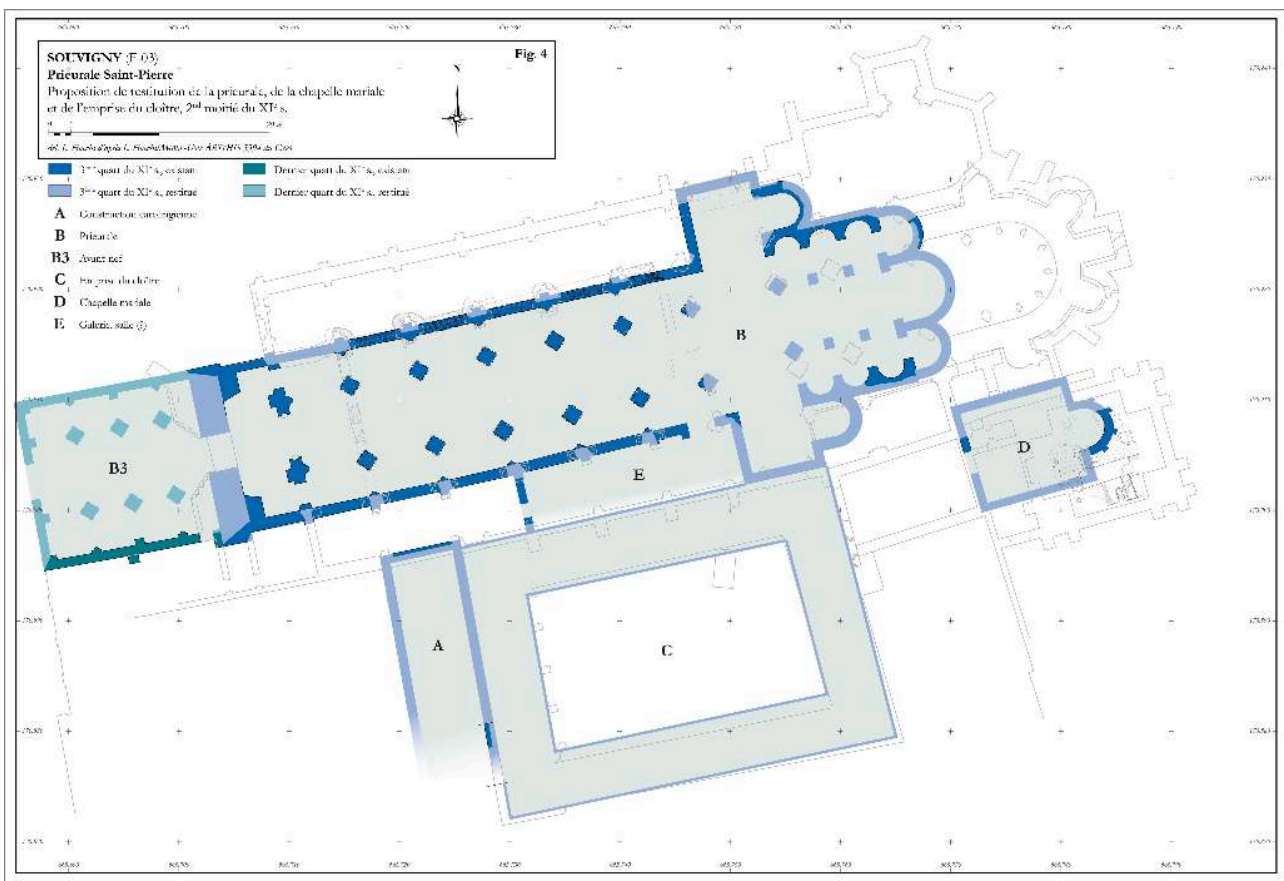

Les joints beurrés caractérisant la campagne d'édification $\mathrm{du} \mathrm{XI}^{\mathrm{e}}$ siècle à Saint-Pierre, apparaissent sur une maçonnerie reprise par la salle capitulaire de la fin du XII siècle, vestige de la façade de l'église de l'Infirmerie de la seconde moitié du $\mathrm{XI}^{\mathrm{e}}$ siècle. La progression et la durée des travaux qui semblent ne pas cesser amènent à poser la question de leur ampleur : faut-il envisager une reconstruction touchant les bâtiments conventuels ou admettre une simple reprise les insérant dans la nouvelle organisation? La reconstruction de Notre-Dame de l'Infirmerie, dans le troisième quart du XI ${ }^{\mathrm{e}}$ siècle, plaide en faveur d'un chantier rationnellement organisé, combinant les exigences du pèlerinage et celles de la vie monastique.

Nous avons réalisé en 2003 un premier sondage destiné à estimer le potentiel archéologique subsistant sous la sacristie du XviII ${ }^{\mathrm{e}}$ siècle où, on le savait, devaient se trouver les vestiges de Notre-Dame-des-Avents, église secondaire mariale illustrant la disposition clunisienne (voir infra). En raison de la déclivité naturelle vers la rivière, le sol de la priorale est situé environ $3 \mathrm{~m}$ plus haut que celui du sol extérieur actuel au sud-est. La sacristie néoclassique est donc surélevée de $3 \mathrm{~m}$ par une pièce voûtée qui servait jusque-là de dépôt à divers objets. Outre les vestiges de Notre-Dame-des-Avents sont apparus ceux, plus inattendus, d'un bâtiment antérieur connu par le texte de la Vita Odilonis rédigée par Jotsald dans le troisième quart $\mathrm{du} \mathrm{xI}^{\mathrm{e}}$ siècle, et démoli pour laisser place à Notre-Dame-des-Avents.

$\mathrm{Au}$ Sud de la priorale de la seconde moitié du XI ${ }^{e}$ siècle, s'étend donc une galerie ${ }^{25}$ puis un cloître probablement quadriportique, avec - au sud-est - près de l'infirmerie et de la salle capitulaire, une chapelle Notre-Dame datable du troisième quart du $\mathrm{xl}^{\mathrm{e}}$ siècle. Il s'agit un bâtiment orienté, de plan simple et de moindres dimensions que l'église principale (fig. 4). Sa nef unique, charpentée, se termine par une abside semi-circulaire saillante, précédée par une très courte travée de chœur, formant un arc triomphal en berceau devant le cul-de-four absidal. Deux tiers de l'hémicycle ont été dégagés, immédiatement sous la dalle en béton qui formait le sol de la salle soutenant la sacristie 
en 2003 (diam. estimé à la corde 3,60 m pour une pf. de 3,40 m ; 1. restituée de la travée droite $4 \mathrm{~m}$ pour $1,95 \mathrm{~m}$ de L.) (fig. 2). L'abside, qui vient entourer celle de l'oratoire antérieur, est extérieurement épaulée par quatre contreforts rectangulaires en moyen appareil soigné de blocs de grès taillés, avec joints épais et lissés à la truelle. Entre les harpages latéraux des contreforts, les bâtisseurs ont utilisé un petit appareil moins ordonné, presque un remplissage en opus incertum, combinant des moellons de calcaire et de grès rouge brisés au marteau proche de celui observé pour la construction de l'avant-nef entre 1070 et 1090. Les matériaux mis en œuvre sont de provenance locale. Le nombre pair des contreforts, dont trois seulement ont été reconnus à l'est et au sud (le troisième au nord-est ayant été en partie détruit), impliquent qu'un nombre impair de fenêtres éclairaient l'abside : soit une baie axiale unique, soit trois.

La largeur restituée de la nef est de 7,40 m, mais seuls $2 \mathrm{~m}$ de longueur du vaisseau ont été dégagés par la fouille, autrement dit son angle sud-est. Deux arcades de sa façade occidentale sont en outre conservées, on l'a vu, prises dans le mur est de la salle capitulaire gothique, ce qui fixe sa longueur dans l'œuvre à 10,80-11 m. L'arc nord offre une ouverture plus grande $(2 \mathrm{~m})$ et se trouve dans l'alignement du chœur de NotreDame. Une porte ouvre vers le sud, elle est conservée dans l'état suivant d'après le plan Évezard. Le chœur est pavé de dalles de grès sur lesquels un aménagement tardif a laissé une série d'empreintes, évoquant une installation liturgique. Le tour de l'hémicycle semble en effet constitué sur $60 \mathrm{~cm}$ de largeur en moyenne d'une maçonnerie différente, qui pourrait marquer la trace d'une banquette presbytérale maçonnée, faisant le tour de la base de l'abside. Cet aménagement d'un type assez archaïque recouvre presque exactement la maçonnerie arasée de l'abside antérieure.

Le sol de la nef est quant à lui fait d'une chape de mortier de chaux fortement chargé en sable gréseux rose saumon, similaire à un des niveaux de sols repérés en 2002 et 2007 autour du premier monuments funéraire des saints abbés dans la priorale (dernier quart du XI ${ }^{e}$ siècle), mais il peut ne pas s'agir ici du sol primitif.

\section{La priorale Saint-Pierre du XII ${ }^{\mathrm{e}}$ siècle, reflet de Cluny III}

La nef de Saint-Pierre est élargie par le percement des murs gouttereaux et le bouchage des baies, puis la construction de collatéraux externes, d'abord au nord, puis au sud. Les travaux progressent alors d'ouest en est. L'édification du grand transept, venu se greffer en partie sur le transept antérieur, marque la fin de cette campagne au début du XII ${ }^{\mathrm{e}}$ siècle.

Quelques années précèdent la construction du nouveau chevet, à partir du milieu du $\mathrm{XII}^{\mathrm{e}}$ siècle $^{26}$. Lorsque le chantier reprend, les travaux débutent à l'est et, comme à Parayle-Monial, on suit leur progression qui enveloppe les murs du chœur du début du $\mathrm{XI}^{\mathrm{e}}$ siècle. La présence de Notre-Dame de l'Infirmerie entrâne un désaxement du nouveau chevet vers le nord-est. Les travaux commencent par la construction de la crypte en couloir annulaire à chapelles rayonnantes, qui rattrape le dénivelé du terrain, suivie, au milieu ou au troisième quart $\mathrm{du} \mathrm{XII}^{\mathrm{e}}$ siècle, par celle du petit transept dont la croisée était couronnée selon la tradition locale par une tour «fine et aérienne ». L'angle sud-est du bras méridional de ce transept oriental vient se coller contre l'angle nord-ouest du vaisseau de la chapelle Notre-Dame. En partie basse de ce bras sud apparaît le seul accès vers la crypte. Supposer l'existence d'une porte dans le 
gouttereau nord de la chapelle permettrait de restituer les possibles circulations de la liturgie stationnale (voir infra et fig. 7).

\section{Notre-Dame des Avents (fin XII siècle) (fig. 5 et 7 )}

Fig. 5 - Proposition de restitution de la priorale, de l'église Notre-Dame des Avents et du cloître à la fin du XII siècle (DAO L. Fiocchi).

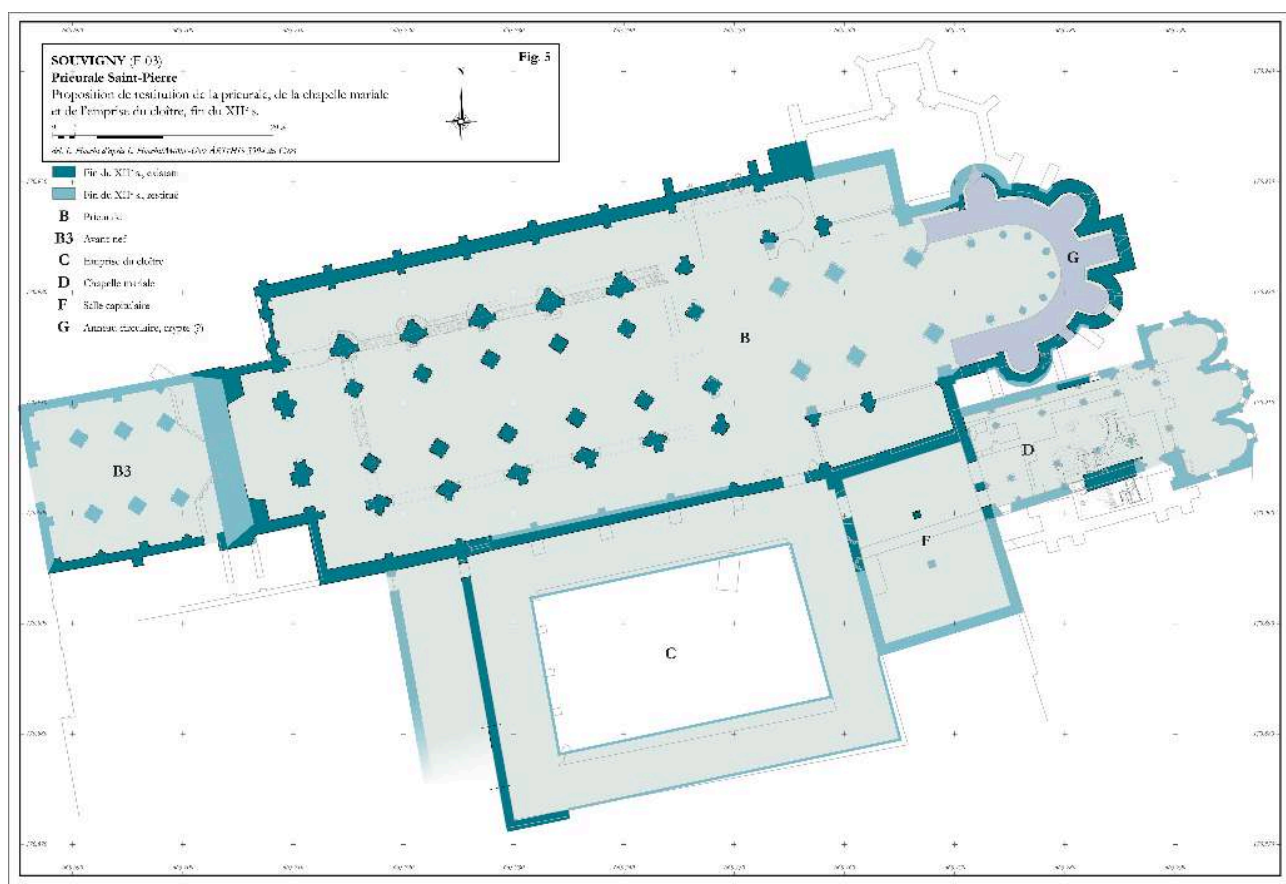


Fig. 7. Plan restitué de Notre-Dame des Avents et des circulations à la fin du XII ${ }^{\mathrm{e}}$ siècle (DAO L. Fiocchi).

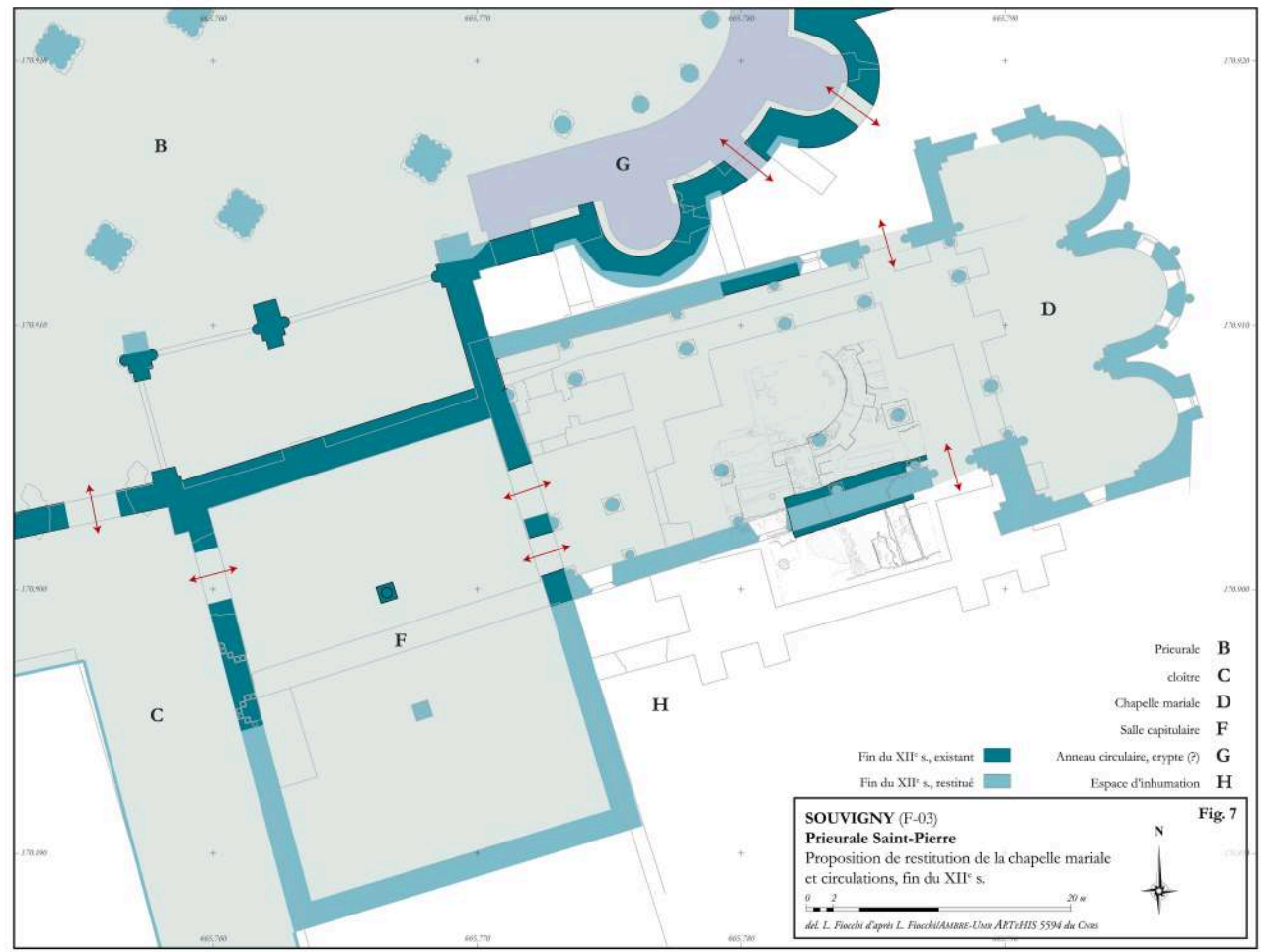

21 L'église romane - connue tardivement sous le titre rare de Notre-Dame des Avents -, enchâssée dans des bâtiments monastiques plus hauts qu'elle et qui laissent sa nef dans la pénombre, "cette vieille chapelle et son précieux sanctuaire, lieu saint où reposent les pères ", est démolie en 1772, pour laisser place à la sacristie néoclassique de l'architecte moulinois Joseph Évezard en 1773-1775. Par chance, Évezard avait pris la peine d'en dessiner plans et coupes en 1769 . 
Fig. 6a - Plan de Notre-Dame des Avents par Joseph Évezard, 1769.

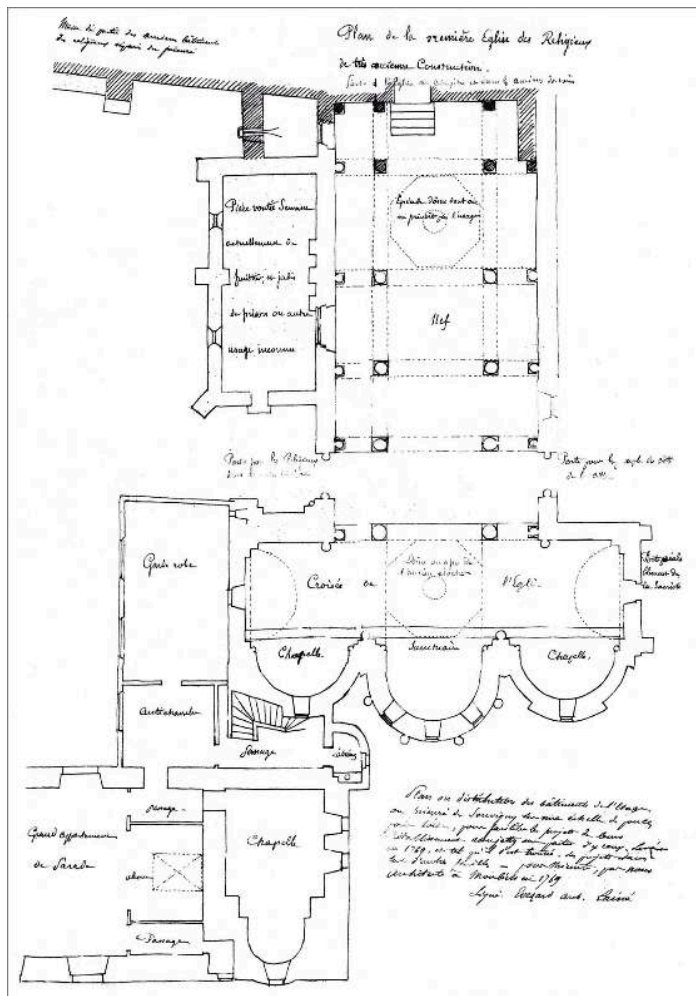

D'après ce " plan de la première église des religieux de très ancienne construction », (fig. 6a) ${ }^{27}$ et le texte de dom Hilaire Tripperet (mi-XviII ${ }^{\mathrm{e}}$ siècle), longue d'environ $20 \mathrm{~m}$ pour environ $7 \mathrm{~m}$ de largeur, Notre-Dame-des-Avents possède un chevet, sans doute roman ${ }^{28}$, à triple abside ouvrant sur un transept débordant, avec coupole sur trompes à la croisée, et une nef à trois vaisseaux de cinq travées assez modifiée ${ }^{29}$. La porte figurée (l'arc le plus large - $2 \mathrm{~m}$ - préservé de l'état précédent comme du reste la façade) ouvre directement sur la salle du chapitre à l'ouest; selon les légendes portées sur le dessin, l'église communique aussi avec les "anciens dortoirs » situés au-dessus, à l'étage. Une annexe, d'allure gothique, la flanque au sud ; c'est au XviII siècle un fruitier. Au sud-est, un ensemble d'adjonctions d'époque moderne relie le chevet à la maison du prieur commendataire, s'étendant au sud. Si cette dernière subsiste encore, les autres bâtiments mentionnés et Notre-Dame-des-Avents ont tous disparus depuis les années 1770. On ne disposait jusqu'en 2003 que d'une superposition hypothétique par Talobre du plan d'Évezard sur le plan actuel ${ }^{30}$, suggérant notamment que le chevet rasé de l'église doit se situer dans la propriété privée voisine sous un bosquet de conifères.

Évezard assoie partiellement sa sacristie sur les maçonneries de Notre-Dame-desAvents, arasées en fondations jusqu'au niveau de l'église de l'infirmerie du $\mathrm{XI}^{\mathrm{e}}$ siècle. La fouille de 2004 a révélé une partie réduite de la nef méridionale de Notre-Dame-desAvents et de l'annexe tardive appelée par lui fruitier: un segment long de 5,15 m du gouttereau sud (ép. 1,20 m), le montant d'une porte ouvrant sur l'annexe sud dans son dernier état, une base complète $(90 \times 90 \mathrm{~cm})$ de la colonnade méridionale de la nef, les fondations de deux autres et de la base de la demi-colonne engagée correspondant à l'une d'elles dans le mur sud. Ce dernier réutilise l'épaulement du chevet du XI ${ }^{e}$ siècle, prolongeant simplement vers l'est le gouttereau sud, où l'on distingue de multiples blocs remployés. Le bas-côté méridional ainsi défini mesure 1,27 $\mathrm{m}$ de largeur, ce qui 
est très étroit mais correspond assez bien au plan d'Évezard. Notons toutefois que nous nous situons au niveau des fondations des bases et que les fût des supports des colonnades, circulaires sur le relevé et la coupe de l'architecte et reposant sur lesdites bases pouvaient être plus étroits de quelques centimètres. Le parement interne du mur était enduit de mortier fin blanchâtre sur l'assise préservée au-dessus du ressaut de fondation. Il ne reste aucune trace des sols de Notre-Dame-des-Avents que les travaux d'Évezard ont fait disparaître. Les deux entrecolonnements attestés en fouille sont effectivement inégaux, comme sur le plan : 1,20 et 1,50 m.

Selon la coupe transversale d'Évezard (fig. 6b), les supports sont des colonnes et des demi-colonnes engagées à chapiteaux feuillagés, les collatéraux sont voûtés en berceau sur doubleaux et le vaisseau central en berceau plein cintre continu sans claire-voie ; une seule toiture en bâtière peu pentue, couverte de tuiles, coiffe l'ensemble et repose sur l'extrados des voûtes.

Fig. $6 \mathrm{~b}$ - Coupe transversale de Notre-Dame des Avents dans la travée précédant le transept, par Joseph Évezard, 1769.

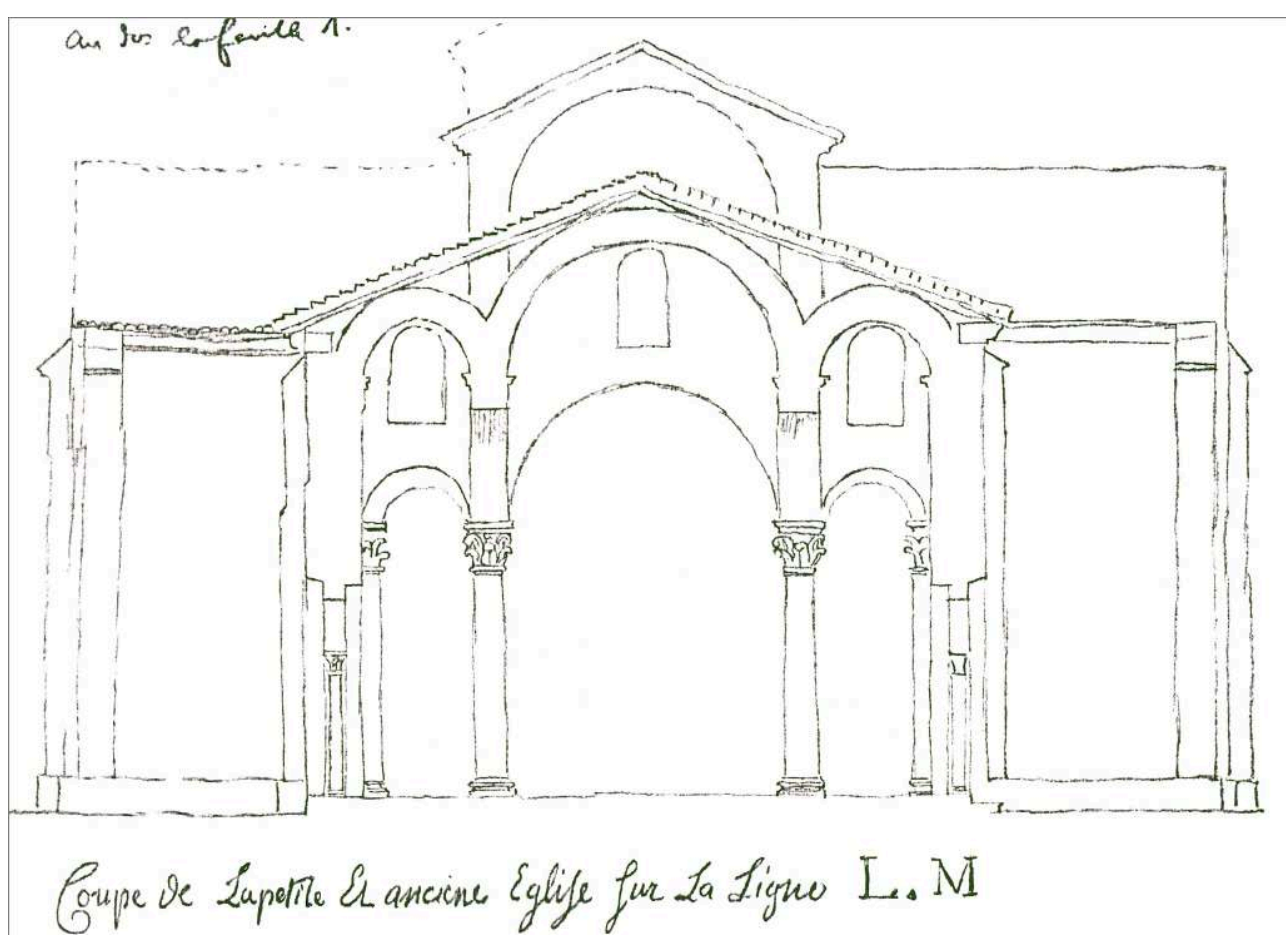

Au nord de la salle voûtée soutenant la sacristie, le gouttereau septentrional de l'église mariale et son bas-côté sont partiellement conservés en élévation sur près de $4 \mathrm{~m}$ de longueur dans une sorte de petit couloir que surmonte à l'ouest l'espace de liaison entre le déambulatoire du XII ${ }^{e}$ siècle de la priorale et la sacristie néoclassique. Celle-ci est en effet presque indépendante de l'église et constitue un pavillon accolé à l'angle sud-est du chœur. Enduit ou au moins badigeonné de l'ait de chaux blanchâtre, le parement interne du gouttereau montre les arrachements de deux colonnes engagées qui soutenaient les doubleaux de la voûte en berceau du collatéral nord. On distingue encore à l'ouest, cette fois sur le mur est de soutènement de la Chapelle Vieille qui joint à cet endroit la façade occidentale de Notre-Dame des Avents, la trace cintrée de la même voûte en berceau et le profil fantôme du même gouttereau. La fouille et l'analyse du bâti confirment tant la faible largeur des bas-côtés $(1,27-1,30 \mathrm{~m} ; 1,25 \mathrm{~m}$ à la 
naissance du berceau) que le voûtement en berceau sur doubleaux (fig. 8) dessiné sur la coupe d'Évezard.

Fig. 8 - Essai de restitution 3D de la nef de Notre-Dame des Avents vue du transept (J. Plantin).

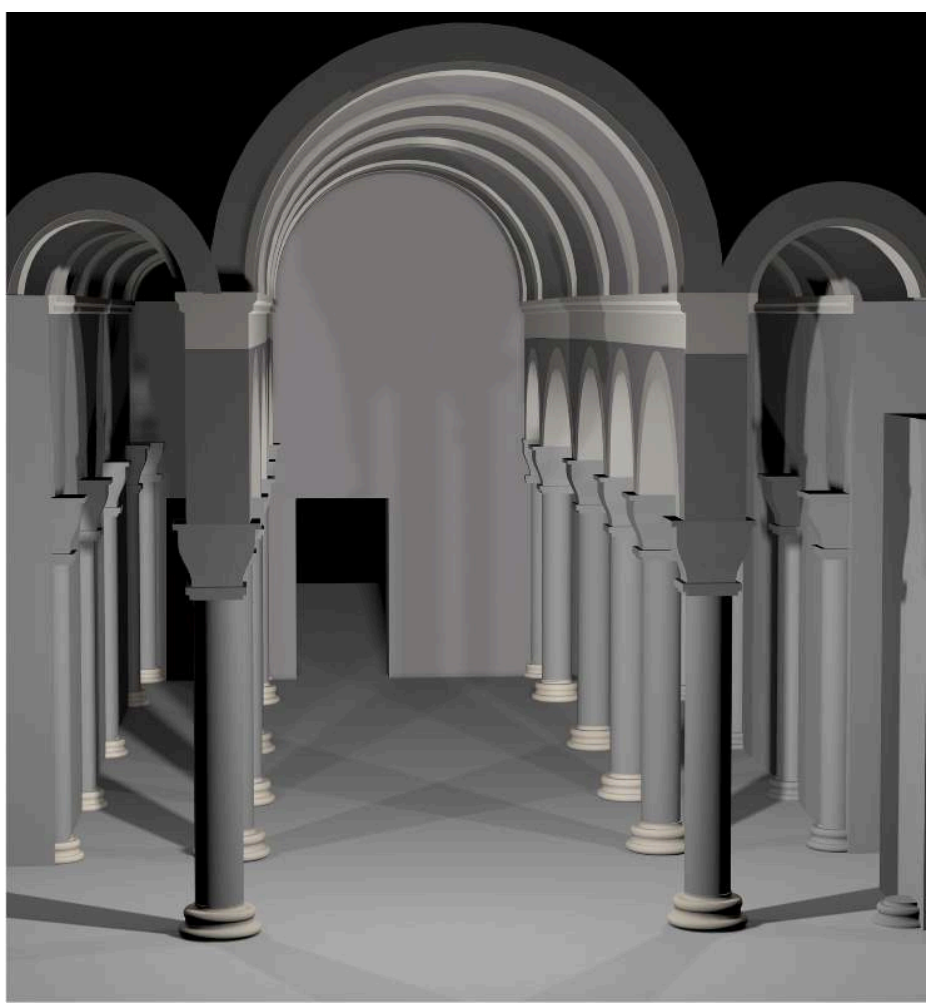

L'examen du plan permet également de voir que la maçonnerie conservée au nord est de la même épaisseur que le gouttereau sud de Notre-Dame de l'infirmerie, repris tel quel sur la longueur de sa nef unique. L'épaississement du mur ne se produit qu'au niveau de l'épaulement de l'abside, ce qui correspond, au nord, à une autre maçonnerie (moderne ?) qui poursuit le couloir vers l'est. Il apparaît donc que les trois premières travées dessinées par Evezard sont installées dans la nef antérieure, tripartie comme on l'a vu faire pour Saint-Pierre dans le troisième quart $\mathrm{du} \mathrm{XI}{ }^{\mathrm{e}}$ siècle. Cette tripartition dans une emprise prédéfinie justifie l'étroitesse des collatéraux et peut-être aussi l'«espèce de dôme dont on ne prévoit par l'usage" (fig. 6a). Il est en effet possible que la division en trois vaisseaux soit intervenue dans un état antérieur à la reconstruction connue sous le vocable des Avents ${ }^{31}$. Cette dernière aurait ensuite simplement développé l'édifice à l'est par une travée voûtée en berceau comme les précédentes selon la coupe transversale d'Evezard (fig. 6b), puis par le transept débordant et la triple abside d'un chevet assez élégant. L'abside centrale était éclairée par trois fenêtres et épaulée par quatre contreforts-colonnes. Une marche marquait l'arc triomphal triple à l'entrée du transept, dont la coupole sur trompes était contrebutée par deux berceaux transversaux, et un double emmarchement surélevait encore le sol de chaque abside par rapport à celui du transept.

Une annexe est accolée au sud de la nef. On ne dispose que d'une portion de son mur est, mais le plan d'Évezard montre des contreforts d'angle obliques d'aspect au mieux gothique. Une partie de la pièce est tardivement pavée de tommettes de $19 \times 19 \mathrm{~cm}$ dont le négatif est assez bien préservé sur près de $2 \mathrm{~m}^{2}$. Ce dernier aménagement peut 
correspondre à la fonction de fruitier attribuée par Évezard à cet espace en 1769. Les couches plus ou moins compactes de démolition de Notre-Dame des Avents reposent immédiatement au-dessus de ce sol soigneusement démonté et dont les tommettes ont dû être remployées ailleurs, comme c'était l'habitude.

\section{Un « plan clunisien » répondant à des exigences liturgiques particulières}

28

« coupoles » l'une à la croisée du transept et l'autre surmontant la deuxième travée de
la nef, entrecolonnements inégaux, triple abside alignée) trahissent une certaine errance dans les choix de plan et de voûtement. Comme le montrent la fouille et l'analyse de bâti, on a voulu préserver certains éléments et s'adapter à l'édifice antérieur: l'enveloppe de la nef de Notre-Dame de l'infirmerie paraît bien être conservée à l'ouest et la maigreur des gouttereaux peut expliquer les désordres de maçonneries et effondrements signalés au XVIII ${ }^{\mathrm{e}}$ siècle.

29

1950, Joseph Talobre estimait qu'il s'agissait de l'église primitive du monastère ${ }^{33}$. En 1988, Walter Cahn ${ }^{34}$ supposait que l'article de Talobre avait servi de base aux réflexions de Kenneth J. Conant sur les plans clunisiens ${ }^{35}$. Le plan de Notre-Dame des Avents (nef à trois vaisseaux, transept et triple abside) est comparable à d'autres églises étudiées par Conant à Cluny, Lewes ou encore Polirone. Effectivement les trois états préromans puis romans successifs de l'église secondaire du prieuré répondent parfaitement au fameux « plan clunisien » restitué par Conant, dont on connaît aujourd'hui les limites.

Les trois vocables de l'église ont tous une valeur mariale évidente : oratoire ou église de la bienheureuse Vierge Marie ${ }^{36}$, puis chapelle Notre-Dame de l'Infirmerie ${ }^{37}$ et enfin Notre-Dame des Avents ${ }^{38}$. Les deux premières titulatures sont identiques à celles rencontrées à Cluny même dans une topographie très comparable. Le dernier vocable mêle deux thèmes liturgiques : la Vierge bien sûr, et l'Avent. En effet, si cette période de quatre semaines qui précède Noël est l'attente de la naissance du Christ mais aussi l'attente eschatologique de la Parousie, elle est pourtant également liée à la Vierge : la naissance du Christ préserve la virginité de Marie ${ }^{39}$. Le culte de la Vierge était très intense dans le diocèse de Clermont et l'abbatiat d'Odilon (qui a d'ailleurs composé des sermons pour les fêtes de la Vierge) correspond à la construction de la crypte de la cathédrale de Clermont dont le chantier date des années 980-1020 ${ }^{40}$, probablement entamé par l'évêque Étienne II (dont Odilon était proche) et destinée à abriter la Majesté de Marie, une statue-reliquaire. L'abbé Mayeul fait un pèlerinage au Puy, autre pôle marial proche ${ }^{41}$. Le vocable de notre église recoupe donc des éléments privilégiés de la spiritualité clunisienne. Mais la titulature complète ne nous est parvenue que par des documents tardifs, puisque la première mention se retrouve dans un texte du 22 décembre 1772 rapportant, lors de la délibération au chapitre, la décision confirmée des moines de détruire "... la vieille chapelle de la Sainte Vierge dite des Avents... » en mauvais état en raison de la démolition d'un pilier ${ }^{42}$. Au XvI ${ }^{\mathrm{e}}$ siècle, Nicolas de Nicolaÿ pour sa part ne mentionnait pas le vocable et se contentait d'écrire « ...sur le derrière de la grande église est l'ancienne église et vieux cloistre audit prieuré. Ledit prieuré est de grand estendue, soit en églises, cloistres, dortoirs, maison prieurale qui est très belle et bien logeable, cours, prés, jardins, celliers; greniers et pescheries et autres maisons des réguliers, le tout [déjà] en grande désertion et ruine à faute d'entretenement des couvertures... » ${ }^{43}$. Le texte de 1772 
indique que la démolition déjà entamée a donné l'occasion " de connoitre la caducité de partie du sanctuaire de ladite chapelle, qui est au midy : caducité qui provenoit premièrement de la destruction ancienne d'un pilier buttant, qui lui était attenant; secondement d'un escalier pratiqué après coup et depuis très longtemps dans l'épaisseur du mur de cette partie du sanctuaire ». L'arc-boutant (gothique ?) peut avoir été supprimé lors de la réunion de Notre-Dame avec la maison du prieur commendataire ${ }^{44}$ - un bâtiment du XvIII ${ }^{\mathrm{e}}$ siècle dont le niveau inférieur est gothique avec un mur de refend peut-être roman. Cette liaison a en effet conduit à percer un porte dans l'absidiole sud du chevet de l'église vers un escalier en vis, ainsi que le montre le plan d'Évezard (fig. 6a).

31 La liturgie de la mort est particulièrement développée dans le milieu clunisien ${ }^{45}$. La mort d'Odilon à Souvigny a été déjà largement évoquée ${ }^{46}$ et l'église Sainte-Marie y joue un rôle de premier plan, car c'est en ce lieu qu'à la fin de l'année 1048, l'abbé Odilon mourant est conduit depuis l'infirmerie proche. La veille de Noël Odilon se rend au chapitre mais le 25 décembre il est transporté dans «l'oratoire de la bienheureuse Vierge Marie »; quelques jours plus tard, la vieille de la fête de saint Sylvestre, à l'heure de Vêpres, "il se fait transporter devant l'autel de Marie, mère de Dieu ", puis il est ramené à l'infirmerie pour prendre du repos dans un petit lit. Il est ensuite déposé sur un cilice, reprenant en cela l'exemple de saint Martin, exemple qui sera suivi ensuite par les abbés de Cluny. Après sa mort, son corps est lavé et parfumé ${ }^{47}$, revêtu de ses vêtements, porté dans la priorale Saint-Pierre sur un brancard et déposé devant l'autel des Saints-Pierre-et-Paul. Selon Bernard, cette pratique est considérée comme relevant de l'ancienne coutume, à son époque elle ne devait plus être en usage.

Selon le Liber Tramitis, à Cluny, la liturgie du dimanche ${ }^{48}$ comporte une procession qui quitte l'église par la porte du bras sud du transept (l'édifice concerné est celui connu sous le nom de Cluny II); la première station a lieu dans la chapelle Sainte-Marie. À Souvigny, la procession doit démarrer dans l'édifice connu vers 1048 sous le nom d'oratoire de la bienheureuse Vierge Marie ${ }^{49}$, puis dans la chapelle de l'Infirmerie reconstruite après 1075 et modifiée à la fin du XII ${ }^{e}$ siècle (sous le vocable de NotreDame-des-Avents). Si l'imitation de Cluny est poussée à son terme, cette procession implique un accès depuis le bras sud du transept oriental et une porte ouvrant dans le mur nord de chacune des deux églises romanes secondaires - à moins bien sûr que l'on emprunte un autre trajet, via le bras sud du transept occidental, la salle du chapitre et que l'on ne rebrousse ensuite chemin. Les maigres vestiges conservés et la topographie monastique souvignyssoise nous amènent à préférer la première option, comme d'ailleurs la logique clunisienne (fig. 7).

33 L'église de la Vierge Marie joue par conséquent un rôle important dans la vie du monastère à Souvigny tout comme à Cluny où elle est non seulement l'édifice marial mais aussi l'église de l'infirmerie. Elle prend également une fonction funéraire de chapelle des morts dès le $\mathrm{XI}^{\mathrm{e}}$ siècle - avec la messe anniversaire de la comtesse Ermengarde, inhumée au chapitre, voir plus haut. Notons que les sépultures n'apparaissent dans l'édifice que tardivement pour ce que nous avons pu en voir. En 1278, Hugues prieur de Saint-Martin-des-Champs (frère de l'abbé Yves II de Cluny qui avait été prieur de Souvigny) est enterré dans la chapelle Notre-Dame de l'Infirmerie, en face de l'autel de la Vierge ${ }^{50}$. Après lui et jusqu'à la Révolution, les inhumations d'officiers du monastère (?) sont nombreuses dans la nef (fig. 2) ; d'autres sépultures en pleine terre attestent la présence d'un cimetière le long de son flanc sud. D'ailleurs, le procès-verbal du chapitre du 2 décembre 1772, indique qu'il a été permis aux moines de 
Souvigny de continuer à démolir le "vieux bâtiment » lors de la diète tenue à SaintMartin des Champs à Paris en septembre 1772, à condition « que l'on préserve 12 à 15 pieds autour pour qu'à perpétuité se trouvent à l'abri de toute profanation les cendres de nos pères qui reposent en ce saint lieu ${ }^{51}$. C'était sans compter les travaux de drainages des Monuments historiques dans les années 1970 puis les fouilles des années 2000.

\section{NOTES}

1. P. CHEVAlieR, « Les tombeaux et les monuments funéraires médiévaux des saints abbés Mayeul et Odilon de Cluny ", in Hortus Artium Medievalium 10 (2004), p. 119-132 ; P. CHEVALIER et A. MAQUET, «La fouille des tombeaux des saints abbés Mayeul et Odilon et les pèlerinages à Souvigny », in Bulletin Monumental 162-II (2004), p. 87-100.

2. P. CheVAlier, M. čaušEvić-Bully, M. Dupuis, L. FIOcChi, O. LAPIE, "Priorale Saint-Pierre de Souvigny, Etude archéologique de la nef, $1^{\mathrm{e}}$ tranche », in BUCEMA 11 (2007), p. 71-77 (http:// cem.revues.org/document1087.html); P. Chevalier, S. BULLY, M. čAUŠEVIĆ-BULlY, M. DUPUIS, L. FIOCCHI, A. BARADAT, «Saint-Pierre de Souvigny. Étude archéologique de la nef, $2^{\mathrm{e}}$ tranche », in BUCEMA 12 (2008), p. 53-59 (http://cem.revues.org/document6382.html).

3. L. FIOCCHI, Le chantier de la priorale Saint-Pierre de Souvigny, $X^{e}-\mathrm{XII}^{e}$ s., M2 d'archéologie, UBP Clermont-Ferrand 2, 2008.

4. P. CheVAlier et A. MAQuet, "Notre-Dame-des-Avents, la seconde église du prieuré de Souvigny ", Nos Églises Bourbonnaises 17, Moulins, déc. 2004, p. 51-65.

5. A. REGOND, «L'architecture du prieuré (XVII ${ }^{\mathrm{e}}-\mathrm{XVIII}^{\mathrm{e}}$ siècle). Sacristie et maison Saint-Odilon », in B. PHALIP, P. CHEVALIER et A. MAQUET (dir.), Souvigny (Allier). La prieurale et le prieuré, Coll. Les Cahiers du Patrimoine 101, Paris, 2012, p. 170-173.

6. A. BERNARD \& A. BRUEL, Recueil des chartes de l'abbaye de Cluny, Paris, 1876-1903, t. 1, n 217, p. 206-207 d'après le Cartulaire A, original perdu.

7. Cf. deux chartes de 950 et 954 : ibid., t. $1, \mathrm{n}^{\circ}$ 783, p. 735-737 d'après le Cartulaire A, original perdu ; et ibid., t. 1, n 871, p. 824-826 d'après le Cartulaire A, original perdu.

8. A. MAQUET, «Cluny et l'Auvergne ", in Odilon de Mercoeur, l'Auvergne et Cluny, Actes du colloque de Lavoûte-Chilhac - 10-12/05/2000, Nonette, 2002, p.291-311; EAD., Cluny en Auvergne, Thèse de doctorat, Université Paris I, 2006.

9. D. MEHU, Paix et communautés autour de l'abbaye de Cluny, $X^{e}-X V^{e}$ siècle, Lyon, 2001. D. Riche, L'ordre de Cluny de la mort de Pierre le Vénérable à Jean III de Bourbon: "le vieux pays clunisien", SaintÉtienne, 2002.

10. L. FIOCCHI, « La priorale et le prieuré ( $\mathrm{X}^{\mathrm{e}}-\mathrm{XII}^{\mathrm{e}}$ siècle) », in B. PHALIP, P. CHEVALIER, A. MAQUET (dir.), Souvigny, la priorale et le prieuré, Cahiers du Patrimoine 101, Paris, 2012, p. 95-98.

11. RAOUl Glaber, Les Cinq livres de ses histoires (900-1044), M. Prou (éd.), Paris, 1886, livre 2-6, 14, p. 41.

12. Le plan de la nef de la seconde moitié du $\mathrm{x}^{\mathrm{e}}$ siècle explique les décrochements de maçonneries des arcades ouvrant sur le bas côté externe sud lors de l'agrandissement à cinq vaisseaux. Ces désordres des parties hautes reprennent en effet en élévation le plan du mur gouttereau de la nef de Mayeul. 
13. Elle est proche de celle édifiée un peu plus tard à Paray-le-Monial : G. ROLLIER, «Premiers résultats des fouilles archéologiques sur la basilique de Paray-le-Monial : les deux prieurales », in Paray-le-Monial, Brionnais-Charolais, le renouveau des études romanes, 1998, Paris, 2000, p. 53-77 ; J.N. BARNOUD, N. REVEYRON, G. ROLLIER, Paray-le-Monial, Paris, 2004. La comparaison avec Souvigny est proposée par C. SAPIN, «Le nouveau plan de Paray-le-Monial et l'architecture du $\mathrm{XI}^{\mathrm{e}}$ siècle en Bourgogne », in Paray-le-Monial, Brionnais-Charolais, op. cit., p. 79-90.

14. On trouve les deux mentions dans la Vita d'Odilon rédigée par Jotsald de Saint-Claude: ecclesia (JOTSALD DE SAINT-CLAUde, Vita Odilonis, J. STAUB (éd.), Hanovre, 1999, p.179) et oratorium (ibid., p. 286).

15. Idée formulée lors de visites du site avec, outre les auteurs, Éliane Vergnolle, Christian Sapin et Sébastien Bully.

16. Cette progression de l'église vers les bâtiments monastiques, avec la mise en place de l'organisation générale du monastère a été observée par Benjamin Saint-Jean-Vitus pour l'abbaye Saint-Philibert de Tournus : B. SAINT-JEAN-VITUS, "Les bâtiments claustraux de Saint-Philibert au Moyen Âge ", Saint-Philibert de Tournus, histoire, archéologie, art, Tournus 1994, 1995, p. 231-248.

17. S. MARCAILle, Antiquitéz du prieuré de Souvigny, Moulins, 1610, p. 225-229.

18. Les psaumes $6,31,37,50,101,129$ et 142 .

19. JOTSALD DE SAINT-CLAUDE, op. cit., p. 182-184.

20. S. CASSAGNeS-BRouquet, "Culte des saints et pèlerinage, Bourgogne, $\mathrm{xI}^{\mathrm{e}}$ au XIII ${ }^{\mathrm{e}}$ siècle ", Les pèlerinages à travers l'art et la société à l'époque préromane et romane, Les Cahiers de Saint-Michel de Cuxa 32, 2000, p. 63-77.

21. C. SAPIN, Le nouveau plan de Paray-le-Monial et l'architecture du XIe siècle en Bourgogne ", Le renouveau des études romanes. Actes du colloque de Paray-le-Monial - 1998, La Pierre-qui-Vire, 2000, p. 79-90.

22. Fouilles menées par René Moreau, publiées par F. DESHOULIÈRES, «Les fouilles de l'église de Souvigny (Allier) », Bulletin Monumental 83, Paris, 1924, p. 160-164.

23. Quinze mètres vingt dans l'œuvre à Souvigny contre $16,50 \mathrm{~m}$ à Cluny.

24. É. VERGNOLLE, "L'ancienne prieurale Saint-Pierre de Souvigny", Congrès archéologique de France, Bourbonnais, 1988, Paris, 1991, p. 399-431; EAD., " Passages muraux et escaliers : premières expériences dans l'architecture $\mathrm{du} \mathrm{XI}^{\mathrm{e}}$ siècle ", Cahiers de civilisation médiévale, $\mathrm{X}^{e}$-XII ${ }^{e}$ siècles, 32, Poitiers, 1989, p. 43-60.

25. Une galerie claustrale de $26,40 \mathrm{~m}$ - bâtiment allongé situé entre la nef et l'aile nord du cloître - se développe du bras sud du transept jusqu'au bâtiment carolingien mentionné plus haut et toujours préservé. Lors de l'élargissement de la nef à cinq vaisseaux le cloître n'est pas reconstruit, ce qui suppose la conservation jusqu'au XIII ${ }^{\mathrm{e}}$ siècle du cloître du milieu du XI ${ }^{\mathrm{e}}$.

26. Cette période de stagnation peut être liée à la crise que connaît Cluny en 1126 avec le schisme dit de Pons de Melgueil.

27. Archives départementales de l'Allier, J9 ; Fr. DESHOULIÈRES, Souvigny et Bourbon-l'Archambault, Paris, 1924, plan p. 73 (peu lisible).

28. W. CAHN, "Souvigny: Some Problems of its Architecture and Sculpture », Gesta, XXVII/1-2, 1988, p. 51-62; É. VERGNOLLE, "L'ancienne prioriale Saint-Pierre de Souvigny », Congrès archéologique de France, Bourbonnais - 1988, Paris, 1991, n. 20.

29. On remarque la deuxième coupole (« dont on ne prévoit pas l'usage »), des entrecolonnements inégaux, une abside nord retravaillée... Un plan au niveau des toitures est également conservé aux $\mathrm{AD}$ de l'Allier (merci à Sophie Liégard pour cette information) : la coupole ouest qui surprend l'architecte s'élève sur un tambour octogonal, alors que celle de la croisée du transept y arbore une toiture en pavillon, par conséquent sur une base carrée.

30. J. TALOBRE, «La sépulture de saint Odilon et l'histoire architecturale de Cluny et Souvigny ", in A Cluny, colloque en l'honneur des saints abbés Odon et Odilon, Dijon, 1950, plan p. 251. 
31. Si l'on admet cette hypothèse, l'arc sud de la façade occidentale pourrait être assigné à cette transformation: il permettrait alors logiquement l'accès au collatéral sud. Suivant la date attribuée à la tripartition de la nef, autrement dit si elle intervenait avant la construction du petit transept, un arc symétrique serait possible au nord. ; il aurait été supprimé à l'édification du bras sud du transept oriental.

32. É. VERGNOLLE, op. cit., n. 20, p. 404, estime que la nef a peut-être été reprise au XVI ${ }^{\mathrm{e}}$ ou au XVII siècle. Voir supra, notre propre hypothèse.

33. J. TALOBRE, op. cit., p. 249.

34. W. CAHN, op. cit., p. 51-62, n. 57.

35. K. J. CONANT, Carolingian and Romanesque Architecture. 800-1200, Yale UP, 1974, p. 196.

36. JOTSALD DE SAINT-CLAUDE, op. cit., p. 179 et 286.

37. En 1278, cf. dom H. TRIPPERET, Mémoires pour servir à l'histoire du prieuré Saint-Pierre-Saint-Paul de Souvigny, BnF, N.A.F. 3602, p. 57v; et M. FAZY, Le livre des anniversaires de Souvigny, Moulins, 1937, p. 24.

38. Nous n'avons trouvé qu'une seule autre appellation comparable: abbé P. LACRoIX, L'église Saint-Christophe à Chissey-la-Loue (Jura), Lyon, sd, p. 17, qui évoque une statue placée à la croisée du transept et appelée jusqu'alors Notre-Dame la blanche qu'il identifie comme étant Notre-Dame des Avents. Merci à Antoine Paillet et Sébastien Bully qui nous ont permis cette comparaison.

39. D. IOGNA-PRAT, E. PALAZzo et D. Russo (éd.), Marie, le culte de la Vierge dans la société médiévale, Paris, 1996 ; particulièrement M. GOULLET et D. IOGNA-PRAT, «La Vierge en majesté de ClermontFerrand », p. 383-405; voir également P.-M. GY, s.v. "Avent », in A. VAUChEz (dir.), Dictionnaire encyclopédique du Moyen Âge, Paris, 1997, t. 1, p. 154-155.

40. P. CHEVALIER, «L'archéologie du bâti appliquée à la révision d'une fouille ancienne, celle de la crypte de la cathédrale de Clermont ", in I. PARRON-KONTIS et N. REVEYRON (éd.), Actes de la tableronde "L'archéologie du bâti : pour une harmonisation des méthodes", Saint-Romain-en-Gal, 2001, Paris, p. 87-94.

41. D. IOGNA-PRAT, Agni immaculati. Recherches sur les sources hagiographiques relatives à saint Maïeul de Cluny (954-974), Paris, 1988, p. 229-232.

42. Abbé P. ChaUdagne, "La sacristie de Souvigny", in Bulletin de la Société d'Emulation du Bourbonnais, 62, 1984-1985, p. 90-96; AD Allier, H 616 bis, p. 78.

43. N. DE NICOLAŸ, Générale description du Bourbonnais, Paris, 1569, rééd. Moulins, 1889, p. 73. Nicolas de Nicolaÿ était le géographe du roi Charles IX, une des quatre versions de son ouvrage était conservée dans la bibliothèque du prieuré de Souvigny.

44. A. REGOND, op. cit., p. 181-182.

45. Voir par exemple D. IOGNA-PRAT, « Les morts dans la comptabilité céleste des clunisiens de l'an Mil », in J.-Ch. PICARD et D. IOGNA-PRAT (éd.), Religion et culture autour de l'an Mil. Royaume capétien et Lotharingie, Actes du colloque Hugues Capet (987-1987). La France de l'an Mil, Paris, 1990, p. 55-70. Repris dans Études Clunisiennes, Paris, 2002, p. 125-151.

46. P. HENRIET, «Chroniques de quelques morts annoncées ", in Médiévales, 31 (1996), p. 93-108; A. MAQUET, Cluny et la mort : le cas des abbés Mayeul et Odilon à Souvigny, in Hortus Artium Medievalum, 10 (2004), p.109-118.

47. Selon les coutumes de Cluny, le corps du moine doit être lavé par les frères avec de l'eau chaude. Voir PL, t. 149, col. 773 ; et C. TREFFORT, L'église carolingienne et la mort, Lyon, 1996.

48. K. KRÜGER, « Monastic customs and liturgy in the light of the architectural evidence: a case of study on processions (eleventh-twelfth centuries) », in S. BOYNTON et I. COCHELIN, From dead of night: The Medieval Customs of Cluny. Du cœur de la nuit à la fin du jour: les coutumes clunisiennes au Moyen Âge, Turnhout, 2005, p. 191-220, en part. p. 195 et suivantes.

49. BHL, 6280 ; PL, t. 142, col. 888-891 ; et J. STAUB, Studien zu Iotsalds Vita des Abtes Odilo von Cluny, Hanovre, 1999 : il a reconstitué la vie de Jotsald, biographe d'Odilon et abbé de Saint-Claude dans 
le Jura. J. Staub a corrigé l'erreur de Mabillon, qui identifiait le destinataire de la lettre des moines de Souvigny comme étant le monastère de Marmoutier alors qu'il s'agit de celui de SaintDenis.

50. Dom H. TRIPPERET, op. cit., p. 57 v ; et M. FAZY, Le livre des Anniversaires du prieuré de Souvigny, Moulins, 1937, p. 24.

51. AD Allier, H 616 bis, p. 78.

INDEX

Index géographique : France/Souvigny, France/Cluny

Mots-clés : église mariale, chapelle des morts, chapitre, infirmerie, Odilon (saint)

\section{AUTEURS}

\section{PASCALE CHEVALIER}

Université Blaise-Pascal Clermont-Ferrand 2 / UMR 6298-ARTeHIS Dijon

\section{ARLETTE MAQUET}

chargée d'enseignement Université d'Auvergne Clermont-Ferrand 1 / UMR 6298-ARTeHIS Dijon

\section{LAURENT FIOCCHI}

archéologue contractuel 